\title{
The Clinical Curative Effect Analysis of Posterior Approach Treatment to
}

\section{Thoracolumbar Fractures}

\author{
Zhongkai Feng ${ }^{1}$, Hua Lu ${ }^{1}$ \\ ${ }^{1}$ Henan Province Hospital of Traditional Chinese Medicine, Zhengzhou, Henan, 450002
}

Keywords: Posterior Approach, Thoracolumbar Fracture, Anterior Approach, Clinical Curative Effect

\begin{abstract}
Objective: The clinical curative effect of posterior approach treatment to thoracolumbar fractures will be researched in this paper. Methods: The object of this research is 60 patients with thoracolumbar fractures treated in our hospital from December 2014 to 2016 March. They were divided into control group $(n=30)$ and observation group $(n=30$ cases) by different treatment methods. In the control group, 30 patients were treated by anterior approach, and in the observation group, the patients were treated by posterior approach. This paper will compare the therapeutic effect of anterior approach and posterior approach. Result: In the observation group, the total effective rate was $96.67 \%$ after the treatment by posterior approach. But in the control group, the total effective rate was $83.33 \%$ after the treatment by anterior approach. There is a significance difference between them, having a statistical significance, $\mathrm{p}<0.05$. The total satisfaction of the observation group was $93.33 \%$ after the treatment by posterior approach, and the total satisfaction of the control group was $76.67 \%$ after the treatment by anterior approach. There is a significance difference between them, having a statistical significance, $\mathrm{p}<0.05$. Conclusion: Posterior approach treatment to thoracolumbar fractures has an obvious curative effect, and it is worthy of popularization and application.
\end{abstract}

\section{Introduction}

Thoracolumbar fracture is a common disease in department of orthopedics surgery clinical fracture. Because of the thoracolumbar spine fractures in patients with limited mobility, this disease has a great impact on the patient's daily life, and greatly reduced the quality of the patient's life[1]. There are two main ways to treat thoracolumbar fractures, posterior approach and anterior approach. For the spine of the patients can be effectively corrected during the posterior approach, and it could effectively reduce the pain of patients as well. What is more, the posterior approach has high security. And its postoperative recovery time was significantly shorter than anterior approach. Therefore, the posterior approach is the first choice for surgery patients. The object of this research is 60 cases of patients with thoracolumbar fractures treated in our hospital from December 2014 to 2016 March. The treatment was performed by anterior approach and posterior approach. This paper will compare the therapeutic effect of them. 


\section{Objects and Methods}

\section{Research Objects.}

The object of this research is 60 cases of patients with thoracolumbar fractures treated in our hospital from December 2014 to 2016 March. All the subjects were consistent with thoracolumbar spine fractures in the relevant literature diagnostic criteria. They were divided into control group (n $=30)$ and observation group ( $n=30$ cases) by different treatment methods. In the control group of patients, there are 13 females and 17 males, the minimum age of them was 22 years old, the oldest was 76, and the median age was (56.4 \pm 5.1 ) years old. There are 18 cases of injury, 4 cases of falling, 8 cases of traffic accidents. In the observation group of patients, there are 12 females and 18 males, the minimum age of them was 21 years old, the oldest was 79 , and the median age was (57.7 \pm 5.8 ) years old. There are 16 cases of injury, 6 cases of falling, 8 cases of traffic accidents. All the baseline date of injury types, age, gender, and other types of studies were processed by statistical software SPSS17.0. The results showed that there was no statistically significant, comparable, P > 0.05 .

\section{Treatment Methods.}

Patients in the control group were treated by anterior approach. The method was as follows: patients should be given a general anesthesia, and then taken supine position. First of all, the fracture position should be given effective positioning measures, after a good location, the posterior part of the spine and the intervertebral disctissue adjacent to the fracture should be resceted. Removing the foreign body around the ligament, then opened the spine, reset the fracture, implanted iliac bone. Finally, a good job of disinfection should be done, cleaned the wound and sew it up, operation ended. In the observation group, 30 cases were treated by posterior approach, The method was as follows: To give patients a general anesthesia, then raised the patient's chest to $10 \sim 15 \mathrm{~cm}$. After giving precise positioning, opened the patient's abdominal, let the spine fully exposed. Exploring the position of the drill hole by using the pedicle of vertebral arch to ensure the drilling position was within the bone wall. And then fixed the pedicle screw effectively, implanted iliac bone at the same time. Finally, did a good job of disinfection, cleaned the wound and sew it up, operation ended.

\section{Statistical Methods.}

Collected and collated the experimental data of this group, All the date were processed by statistical software SPSS17.0. The count data and measurement data were used $n \%$ and $(x+s)$ seperately. The differences between groups by $\mathrm{x}^{2}$ and $\mathrm{t}$ test ,in $\mathrm{P}<0.05$, the difference had statistically significant.

\section{Results}

\section{Comparison of Clinical Therapeutic Effects in All Patients.}

In the observation group, the total effective rate was $96.67 \%$ after the treatment by posterior approach, and in the control group, the total effective rate was $83.33 \%$ after the treatment by anterior approach, There is a significance difference between them, having a statistical significance, $\mathrm{p}<0.05$. The results were shown in table 1 . 
Table 1 Comparison of the clinical therapeutic effects of all patients [n (\%)]

\begin{tabular}{cccccc}
\hline groups & cases & Effect(n) & Effective(n) & Invalid(n) & Total effective(\%) \\
\hline observation group & 30 & $17(56.67)$ & $12(40.00)$ & $1(3.33)$ & 96.67 \\
control group & 30 & $12(40.00)$ & $13(43.33)$ & $5(16.67)$ & 83.33 \\
$X^{2}$ & & 5.5639 & 0.2281 & 9.8864 & 9.8864 \\
p & 0.0183 & 0.6329 & 0.0016 & 0.0016
\end{tabular}

\section{Comparison of Patients' Satisfaction with Surgery in All Patients.}

The total satisfaction of the observation group was $93.33 \%$ after the treatment by posterior approach, and the total satisfaction of the control group was $76.67 \%$ after the treatment anterior approach, there was a significance difference between them, having a statistical significance, $\mathrm{p}<0.05$. The results could be shown in table 2 .

Table 2 Comparison of patients' satisfaction with surgery [n (\%)]

\begin{tabular}{cccccc}
\hline Groups & Cases & Very satisfied(n) & Satisfied(n) & Dissatisfied(n) & Total satisfaction(\%) \\
\hline observation group & 30 & $16(53.33)$ & $12(40.00)$ & $2(6.67)$ & 93.33 \\
control group & 30 & $9(30.00)$ & $14(46.67)$ & $7(23.33)$ & 76.67 \\
$X^{2}$ & & 11.1969 & 0.9059 & 10.8845 & 10.8845 \\
$\mathrm{p}$ & 0.0008 & 0.3412 & 0.0009 & 0.0009 \\
\hline
\end{tabular}

\section{Discussion}

Thoracolumbar fracture is a common disease in department of orthopedics surgery clinical fracture. Because of the thoracolumbar spine fractures in patients with limited mobility, this disease has a great impact on the patient's daily life, and greatly reduced the quality of the patient's life ${ }^{[2]}$. Nowadays, patients with thoracolumbar fracture increase continuously caused by traffic accidents, fights and construction. Due to the special position of thoracolumbar spine, just in the turning point of lumbar kyphosis and fixation, lumbar lordosis and activity place, local internal stress is relatively concentrated, harmful violence or external force can cause spinal cord injury and combined injury of thoracolumbar fractures, let the injured spine of the patients loss bearing capacity. After the injury, due to nerve compression, patients will have lower limb restriction and other movement disorders, what is worse, the patient would be paralyzed ${ }^{[3]}$. It will cause serious damage to the life of the injured, lossing their normal lives ${ }^{[4]}$.

In the clinical treatment of thoracolumbar fractures, the injured were treated with posterior approach mainly because it can carry on the effective reduction to the fracture location of the injured person, then effectively reduce the pain of the injured. At the same time, the amount of bleeding was less by posterior approach, avoiding the occurrence of major bleeding and other dangerous situations. The posterior approach has a significant effect, it is the preferred method of treatment of thoracolumbar spine fractures ${ }^{[5]}$. This study shows that in the observation group, the 
total effective rate was $96.67 \%$ after the treatment by posterior approach. But in the control group, the total effective rate was $83.33 \%$ after the treatment by anterior approach. There is a significance difference between them, having a statistical significance, $\mathrm{p}<0.05$. The total satisfaction of the observation group was $93.33 \%$ after the treatment by posterior approach, and the total satisfaction of the control group was $76.67 \%$ after the treatment by anterior approach. There is a significance difference between them, having a statistical significance, $\mathrm{p}<0.05$. It shows that the curative effect by posterior approach is good. At the same time, the high satisfaction of the operation has a good agreement with the study of Long gang $\mathrm{Xia}^{[6]}$.

In short, the thoracolumbar spine fracture were treated by posterior operation has an obvious curative effect, it is worthy of popularization and application.

\section{References:}

[1] Miao Zhenlin. 44 Effect Observations of the Treatment of Thoracolumbar Fractures with Posterior Operation [J]. Clinical Medicine of China, 2014, 30:8-10.

[2] Zheng Ganxuan, Lin Weiguang, Hu Yishan. Effect Comparison of the Treatment of Thoracolumbar Fractures between Posterior Approach and Anterior Approach with Loading Sharing Score $\geq 7$ [J], Guangdong Medical Journal, 2014, 35:2084-2087.

[3] Li Riwang. The Curative Effect Analysis of the Treatment of Thoracolumbar Fractures with Posterior Operation [J], Hebei Medical Journal, 2013, 19:710-713.

[4] Lv Haiwen. The Curative Effect Analysis of the Treatment of Thoracolumbar Fractures with Posterior Operation and Bone Graft Operation [J], Clinical Medicine, 2016, 36:75-77.

[5] Wen Jian. The Clinical Effect of the Treatment of Thoracolumbar Fractures with Posterior Operation [J], Gansu Medical Journal, 2016, 35:429-430.

[6] Xia Longgang. The Curative Effect Analysis of the Treatment of Thoracolumbar Fractures [J], Journal of Clinical Medicine Literature, 2016, 3:71-72. 